\title{
Software Quality Assurance in Internet of Things
}

\author{
Marwah \\ Department of Software \\ Engineering, Fatima Jinnah \\ Women University Rawalpindi, \\ Pakistan
}

\author{
Qudsia Mateen \\ Department of Software \\ Engineering, Fatima Jinnah \\ Women University Rawalpindi, \\ Pakistan
}

\author{
Mehreen Sirshar \\ Department of Software \\ Engineering, Fatima Jinnah \\ Women University Rawalpindi, \\ Pakistan
}

\begin{abstract}
Internet of Things (IoT) is increasing the connectedness of things and people on a scale that was not imaginable before. The pace of adoption of IoT in market is increasing due to ubiquitous computing and increase in the connectedness of machines and people. But very few parameters exist to check and ensure the quality of IoT implemented devices and machines. The paper is the comprehensive survey of techniques and methodologies used for the implementing IoT. Based on the defined criteria of evaluation, we have done analysis and compared techniques and have finally concluded that most of the IT implementation techniques lack tool support. Because of the increasing use of IoT, an increasing trend towards its implementation in real time systems has also been observed. The paper assesses strong and weak points of techniques and draws a conclusion at the end.
\end{abstract}

\section{General Terms}

Survey, Quality Assurance.

\section{Keywords}

Internet of Things (IoT), OPred, Round Trip Time (RTT).

\section{INTRODUCTION}

While developing any product and services it is important to assure the quality of product in order to increase its market value. The quality assurance is the systematic way to check whether the product and services are meeting the specified requirements and standards, achieving the desired level of satisfaction of the customer. The consequences of quality assurance increase the customer confidence, satisfaction, credibility of the product and enable the product to compete with other products in the market.

The Internet of Things (IoT) represents a revolution happening now-a-days which results in increased intercommunication and autonomous machine-to-machine data transfer. But with great opportunity comes great responsibility. Along with its conveniences, the IoT unveils several security challenges: in data privacy, safety, governance and trust.

S. Jieet addresses the issues of IoT in web based surveys [1].Fagen $\mathrm{Li}$ and Pan Xiong addresses the security issue of IoT, G. M. Foody, and D. S. Boyd addresses and resolves issues in using volunteered data in land cover map validation [2], T. H. Szymanski presents the cost challenges in IoT. To address all these problems, the paper surveys various IoT implementation techniques. These techniques are then evaluated on different parameters and at the end, conclusion is drawn.

The rest of the sections in the paper are as follows: Section 2 gives the existing implementation techniques in detail. A comprehensive analysis of the techniques is presented in Section 3. Section 4draws conclusion at the end.

\section{SOFTWARE QUALITY ASSURANCE IN INTERNET OF THING}

Internet of Things (IoT) is technological wave in information technology after the Internet. The IoT connects every physical entity of the world with the internet for switching information and communicate with other entities. It is used to recognize, trace, follow, observe and supervise things. It extends and expands the communication between human and human, or human and machine, or machine and machine where machine can be any physical entity. IoT is achieved through RF identification (RFID) and sensor technology.

The survey provides a deep knowledge of quality assurance standards and their comparison. An evaluation criterion is defined in table 1, for comparison. Various related quality assurance techniques have been discussed.

\subsection{Effect of Incentives On Web-Based Surveys (S. Jieet al., 2008) [1]}

This area focuses on discovering the effects of incentives on traditional surveys and to increase the response rate, maintenance rate, and response quality of surveys. Different incentives including material, non-material, monetary, prepaid, promised, conditional, unconditional incentives are used to study the effects on the surveys. In traditional surveys, nonmaterial, pre-paid and unconditional incentives are more efficient. Whereas in web based surveys, material incentives are more effective. Bonus points as compared to gifts and cash lotteries increase the response, maintenance rate and speed of the survey. Prepaid incentives boost the response rate and lower the cost of the survey. Non material incentives on web surveys have a negative impact shrinking the quality of the survey. Incentives given at the start of the survey are proved to be more successful.

\subsection{Volunteered Data in Land Cover Map Validation: Mapping West African Forests} (G. M. Foodyet al., 2013) [2]

Accuracy is the main concern of the project which is of great use in mapping land covers. But this is hindered by absence of quality ground reference data. Volunteers contribute a lot and their data is of the concern that exact data can be achieved. Assessment of glob cover map of tropic forest in West Africa can be supported by Internet based ground photographs. There are four volunteers who detect 1 value in presence of forest and 0 in absence of forest and each value have a label. Each label is compared with other. These labels are then forwarded to reach on a contract between volunteer's reference data and glob cover map. The data is proven to be beneficial and detailed data along with the class labels for validation. Although volunteer's labels and glob cover maps have conflicts but approximation of forest cover was almost exact to that of authoritative maps. 


\subsection{Generalization Evaluation of Machine Learning Numerical Observers for Image Quality Assessment (M. M. Kalayeh et al., 2013) [3]}

This paper proposes two Numerical Observers (NO) in replacement to human observer. Machine learning forms the basis for these NO to detect image quality. Aim of NO is to guess the human observer presentation in heart pouring perfusion-defect protection task which is used for Single Photon Emission Computed Tomography (SPECT) images. In this approach, human observer's score is model for a given image by a degeneration function of a feature derived from the image. The Channelized Hotelling Observer (CHO) is most popular NO used. But the proposed NO has higher accuracy than $\mathrm{CHO}$ along with decreasing the model complication and time taken for computation. It inherits design plainness with lower cost of machines and increases the result time 500 more times. This NO has a disadvantage that it requires more skilled human observers.

\subsection{Optimization of the Deployment of Temperature Nodes based On Linear Programming in the Internet of Things $(\mathrm{L}$. Hu, Z. Zhang et al., 2013) [4]}

This emphasizes on reducing the energy consumption in sensor networks. This area focuses to arrange sensor nodes to document data of temperature and to calculate temperature of sensor nodes using linear programming algorithm in Matlab for a better quality of nodes. Predicting of temperature is done through different steps. Firstly nodes are arranged, and data is collected and analyzed along with historical data. Data is then separated into two groups of training and testing. Then calculation of value and confirmation of the limitation is done with the help of different equations. The temperature is thus predicted and lastly compared with the tables and output figures. This technique enables to decrease the sample rate and optimize the node consumption to lessen the energy and temporary sensor failure detection .Data calculation of sensor temperatures results in that number of sensors can be decreased. It also reduces sensor network energy power consumption along with the node cost and the number of battery substitutions.

\subsection{A Paradigm Shift in Internet-based}

Remote Experimentation (R. Bose, 2013) [5]

This area addresses the issue regarding deficiency of good lab facilities and skilled instructors. To solve this problem experimentation from far off areas is made possible. The method and practice of teaching are student centered processes. Thus virtual labs which are internet based are being made to control the quality. Internet based virtual labs provide all services online and user can only view the front end which he operates. It solves the problem of students at far off areas which have deficiency of resources. It simulates the interests of the students and allows them to acquire knowledge in their own areas. It provides access to utilize the resources and provide a complete learning management system. For assessment techniques, feedback from user is needed. This methodology is a profit based method because of open educational platform. Thus it enrolls more students and is available for more hours and week days and at weekends.

\subsection{Semantic Interoperability Architecture for Pervasive Computing and Internet of Things (J. Kiljander et al., 2014) [6]}

Pervasive computing and internet of things are revolutionizing [6] these days. To cater the interoperability between different functionalities of IoT, an iterative semantic design has been proposed. It uses semantic procedure which is used to increase the quality when IoT and pervasive computing are used together. The main ideas of this architecture are that information and capabilities are represented with semantic web knowledge. Also global IoT is divided into local smart spaces which are maintained through Semantic Information Broker (SIB). Vital idea of architecture is the resolution framework to solve the addresses of SIB either by utilizing the physical object identifier as pointer or by finding it as a discovery parameter. This provides basic information and help for IoT developers. But this also needs tools which stands in favor of improvement and employment of application in future work. Evaluation can be done through performance studies which tell about suitability and validation of architecture.

\subsection{Quality Assessment of Despeckled Sar Images(S. G. Dellepianeet al., 2014) [7]}

This paper focuses on quality detection of noise reduced Synthetic Aperture Radar (SAR) images. Statistical methods do not provide exact quality of SAR images. Statistical methods are used to detect the filter quality but these methods require noise free images. This is applicable only in virtual images but not for real time which have noisy images. Statistical analysis does not provide any information about which filter is the best in performance measures. Frequency domain analysis is used to tackle this problem of quantitative analysis. The most important evaluation is the visual quality judgment which is further used as a reference for qualitative analysis. A new approach is introduced to select best filters for noise reduction, edge and texture conservation in the SAR images. It achieves the best results of filters and is applicable to any filter method. It overcomes the problem of existing approaches.

\subsection{Practical Secure Communication for Integrating Wireless Sensor Networks into the Internet of Things (F. Li, 2013) [8]}

This area put stress to produce a secure scheme for embedding a wireless network into internet of things for improving the quality. This commences an association of a channel between a sensor node [8] to send data and internet host to receive data. For public key validity, two frameworks are available. Public key Infrastructure (PKI) is at the internet host end. It provides a secure link between key and identity of the user and supplies certificate authority. But all record along with the validation of the certificates is to be managed. However, Identity Based Cryptography (IBC) is at sensor end. It provides a unique address to the user and eliminates the need of certification. This scheme attains secrecy, reliability and verification the communication. Sensor node is proficient of identity based secrecy [8] to send a message to the host. The scheme divides sign cryption in two states. One state is offline in which heavy data processing is done. Other state is online in which light data processing is done along with the message. IBC is suitable for small networks and PKI is suitable for internet. 


\subsection{Internet of Things for Smart Cities (A.} Zanellaet al., 2014) [9]

This paper focuses on IoT system development in urban areas. These are characterized by using specific application domain. This is designed to utilize advance communication technologies to stand by the services of admin and residents. It increases the quality of services being offered. Smart city services include control of traffic congestion, waste management, energy consumption, lightning and automation in public buildings .etc. IoT system has a compact architecture in which different secondary procedures are implemented over urban areas. It generates variable data which is then transferred through communication lines and stored and being processed. Internet Engineering Task Force (IETF) web based design for IoT requires implementation of protocols in different layers. It has three layers, data, application, transport and network. etc. IoT for smart cities is helpful in many ways. It gives better use of public resources, reducing cost of public admin, maintaining public services, awareness among citizens. IoT has a drawback that business model is still missing.

\subsection{An Efficient Multi-Dimensional Fusion Algorithm for IoT Data based on Partitioning (Jin Zhou et al., 2013) [10]}

To establish an adequate quality of intelligence, diverse and capacious data have to merge together. It introduces a proficient enumeration method called as data fusion algorithm which deals with data to improve efficiency. The basic idea of this algorithm is that a large data set of higher magnitude is divided into blocks of lower magnitude set which can the easily be practiced. Working out for the core characteristic is done which sets all data subsets to establish a global set. Reduction calculation for global data is resolute and extracts the relations of multi-dimensional data. This algorithm provides a great advantage of renovating big problems into smaller ones by reducing sequential and contiguous complication in data fusion. Applications of data fusion are interoperability and incorporation of diverse data, data abstraction and access, examining search and discovery, semantic reasoning and interpretation and data management and mining.

\subsection{Guest Editorial: Special Issue on} Wireless Sensor Networks, Cyber-Physical Systems, and Internet of Things (X. Mao et al., 2011) [11]

IoT is facing many challenges due to huge number of Wireless Sensor Networks (WSN) which are being used to implement IoT. Since each sensor is connected to some battery having some degree of energy, hence constructing a life time sensor network is a challenge. Sensors are required to be allocated efficiently because of limited bandwidth and huge hindrance of other wireless networks. The area of coverage of sensors, time synchronization and security issues in WSN are resolves by using 'Travel Plane' software project which is based on three algorithms which use searching techniques to resolve the coverage issue of WSN.

\subsection{A Vision of IoT: Applications, Challenges, and Opportunities with China Perspective (S. Chen et al., 2014) [12]}

IOT is facing many application and quality standard problems these days so a three layered platform agenda is proposed in China. The three layered platform architecture involves, sensing layer, business layer, application layer. Sensing layer is used in standard interface and protocol. Hardware modularization, common interface of sensors, intelligent working and self adaption of modules are important characteristics of this platform. Business layer is implemented in resource and administration platform. It provides data management capabilities which are used in various IoT applications. It provides network connectivity functions as well. Application layer is a part of open application platform. Various applications can be published to this platform and users can get application information and subscribe applications through this. Hence it is the way to provide flexible application environment for IoT.

\subsection{Image and Video Quality Assessment Using Neural Network and SVM (D. Wenruiet al., 2008) [13]}

Images and videos are compressed at sender side before transmitting and are reconstructed at receiving end hence there is a need to check their quality before and after transmission. Structure Similarity (SSIM) method is used to assess similarity in structure of image and video before and after transmission. The image is firstly divided into neural network and support vector machine, then, SSIM and Peak Signal to Noise Ratio (PSNR) are used to check the image quality. The video quality is assessed by taking mean value of absolute differences in all scenes. The results show that this method reflects better quality of image. However the Human Visual System (HVS) features must be evaluated in this method in order to get even better results.

\subsection{Max-Flow Min-Cost Routing in a Future-Internet with Improved QoS Guarantees ( T. H. Szymanski, 2013) [14]}

Maximum flow minimum cost algorithm computes cost by fixing maximum flow in specific area. It calculates bandwidth energy and bandwidth distance capacities in one way traffic within an area. It actually needs two linear programs (LPs) to do all computation. The first LP is used to get the highest possible quality flow between various internet connected entities (things), while the second one reduces the cost by considering fixed maximum flow. Hence quality is assured not only by maximum flow but also by minimum cost. Multipath routing cost is calculated by defining its constraints which reduce the LPs and result in faster transmission with high energy efficiencies.

\subsection{An Online Performance Prediction Framework for Service-Oriented Systems (Y. Zhang et al., 2014) [15]}

Web services are software systems designed to support machine to machine interaction over the network [15]. High quality systems are urgent in ever increasing demand of web services. Their performance is highly affected by web services. Hence it is required to predict the performance of such systems. Since the web services depend upon the service status and network environment which vary with time, hence the systems' performance is measured with respect to time. These requirements are met by using an online performance prediction framework, OPred. OPred makes predictions by analyzing past experiences. It builds models and employs time series analysis techniques. However prediction accuracy is not 
checked by OPredand so, data smoothing, information about contents of data is not verified by using OPred.

\subsection{Voip Quality Of Service In Networks With Active Queue Management (V. A. Reguera et al.,2009) [16]}

Quality of Voice on Internet Protocol (IP) Applications (VoIP) and the effect of queue management in networks upon these services is measured by Active Queue Management System (AQMS). This system is used for predicting quality of service in voice applications. Simulation is done on computers and results are compared with the previous results of AQMS. It is found that the results in both cases are same AQMS has lower cost. The system is also applicable for design, sizing and configuring VoIP systems. It also allows study of interaction of various devices with network where IoT technique is implemented, shortens time of simulation and low cost results. Hence active queue management is better than the traditional queue techniques used.

\subsection{7) Trustworthy sensing for public safety} in cloud-centric internet of things (B. Kantarciet al., 2014) [17]

Sensing as a service is the service which enables access to IoT. IoT's quality can be enhanced in smart phones. Cloud computing concept is used in many smart phones and other IoT devices. Sensing-as-a-Service (S2aaS) scheme enables IoT access. This scheme is termed as Trustworthy Sensing for Crowd Management (TSCM). TSCM gathers required sensing data based on cloud computing and then uses algorithms to select devices which will collect data. Then it determines payments to the mobiles if devices being used are the mobile phones. Evaluation of results shows that TSCM enhances public safety effectiveness up to $85 \%$. One of the greatest advantages of TSCM is that other models can be implemented with it in order to increase their efficiency.

2.18) Communicating power supplies: Bringing the internet to the ubiquitous energy gateways of electronic devices ( $S$. Lanziser et al., 2014) [18]

The great issue of energy consumption can be reduced only if the user knows energy consuming device and amount of energy being used by it. The idea of 'Communicating power supply' (CPS) answers these questions by identifying energy consuming appliance and reducing energy utility. It sets a communication between device and its energy consumption.
By using the idea of IoT in each device, energy utility can be reduced by CPS. Some plug loads are also available to identify and control energy consumption but those plug loads require the energy using appliance to be switched 'on' all the time. Secondly no unique identity is given to each device in those loads. However, CPS monitors energy only when the appliance is switched 'on' and it assigns a unique Internet Protocol (IP) to each device for easy identification. This easy to configure and low cost idea is going to become an excellent IoT application in future.

\subsection{9) RTT Prediction in Heavy Tailed Networks (L. R. Dominguez, 2014) [19]}

Quality of transmission in Transmission Control Protocol (TCP) is enhanced significantly by decreasing Round Trip Time (RTT) of packet which has to be sent. RTT predictor algorithm is used to predict the RTT value before sending the packet. If predicted value (known as Retransmission Time Out (RTO)) is less than actual value of RTT, the packet is sent by changing the bandwidth because the packet may $b$ lost otherwise. If RTO is greater than RTT, then packet may take extra time to be sent. Hence RTT accurate prediction is necessarily required, not only for transmission from system to system but also for communication between various devices connected through IoT. The values of algorithm are compared to previous results and found to be more beneficial and less complex with less error value.

\subsection{0) A Priced Public Sensing Framework for Heterogeneous IoT Architectures (A. E. Al-Fagih et al., 2013) [20]}

Billions of devices are linked with internet constituting the IoT which are connected to wireless sensors. But the large number of sensors result in special issues in IoT. For example, price issue, resource management and inter operability etc. These issues are resolved by Priced Public Sensing (PPS) framework, which gives better quality of service by ensuring price control, less data delays and better delivery ratio. PPS is based on n-tier architecture because it is implemented on heterogeneous sources of data. In this framework, access points act as starting points for user requests. Then algorithms are implemented to ensure delay sensitive transmission and at the end, results are calculated which show better performance in terms of less delay, better delivery ratio of packets and less data prices than previously used techniques [20]. But PPS drawback is that it is implemented on mobile phones, radios, vehicles without considering their accurate paths of motion.

Table 1: Evaluation Parameters for Quality Assurance

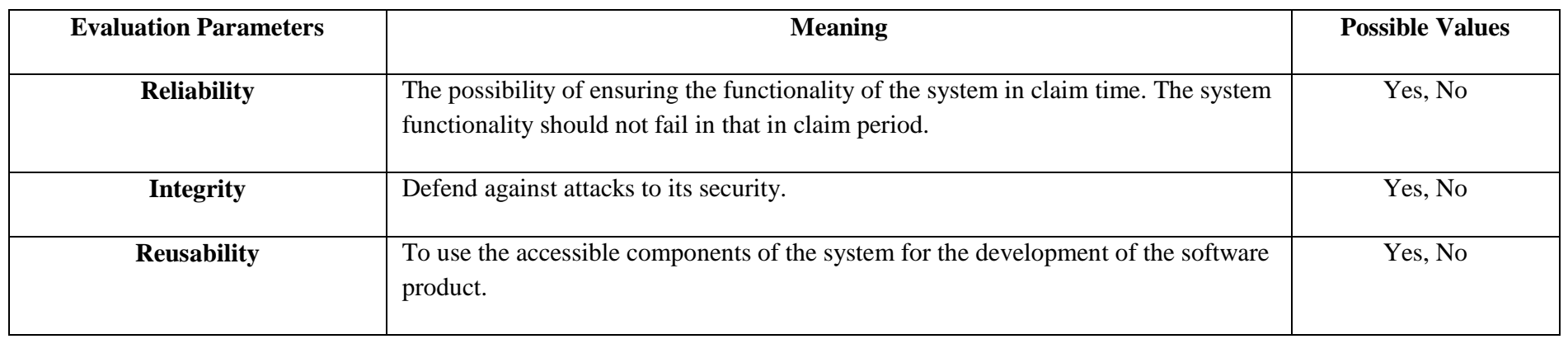




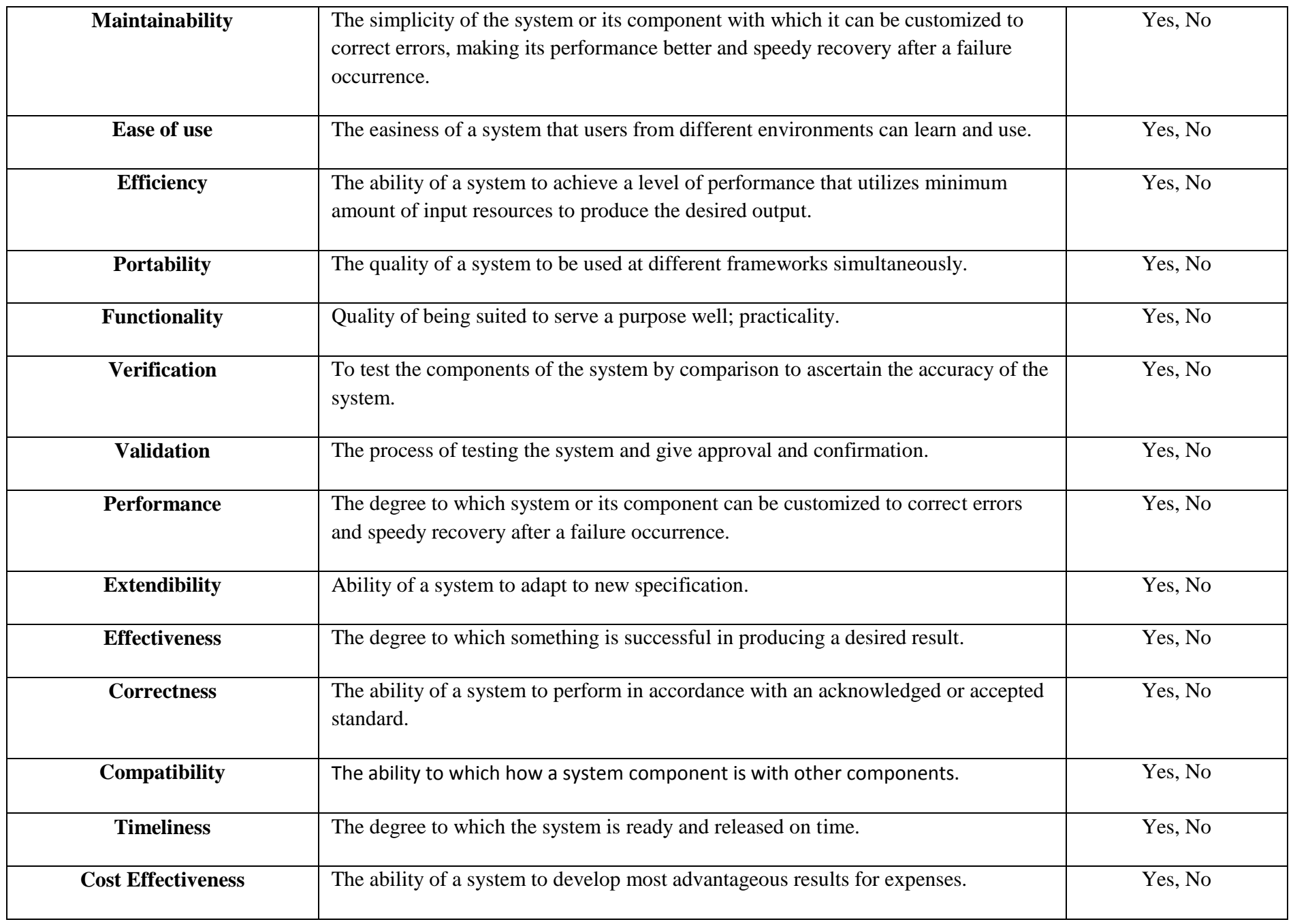

Table 2: Analysis of Quality Parameters

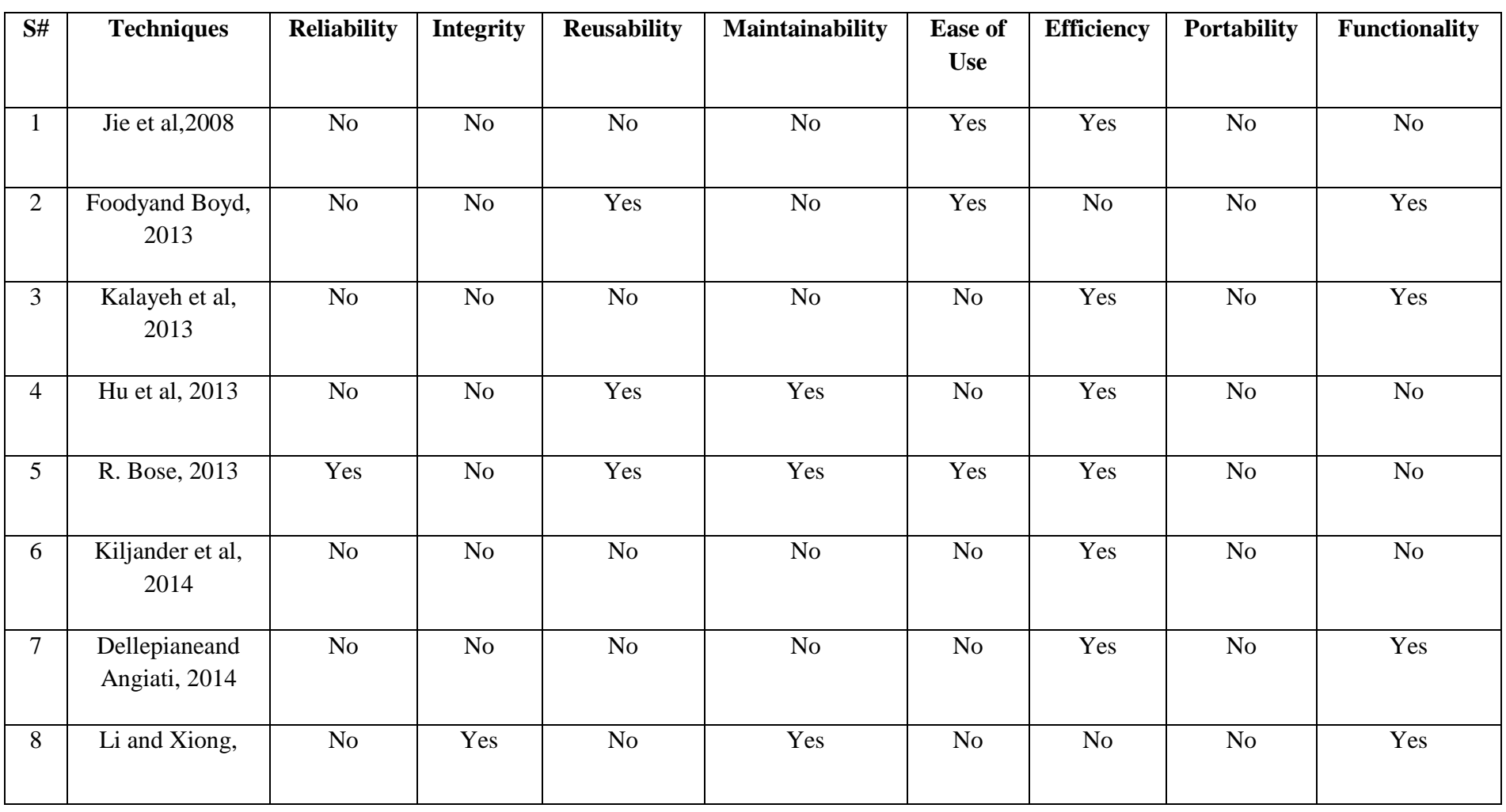




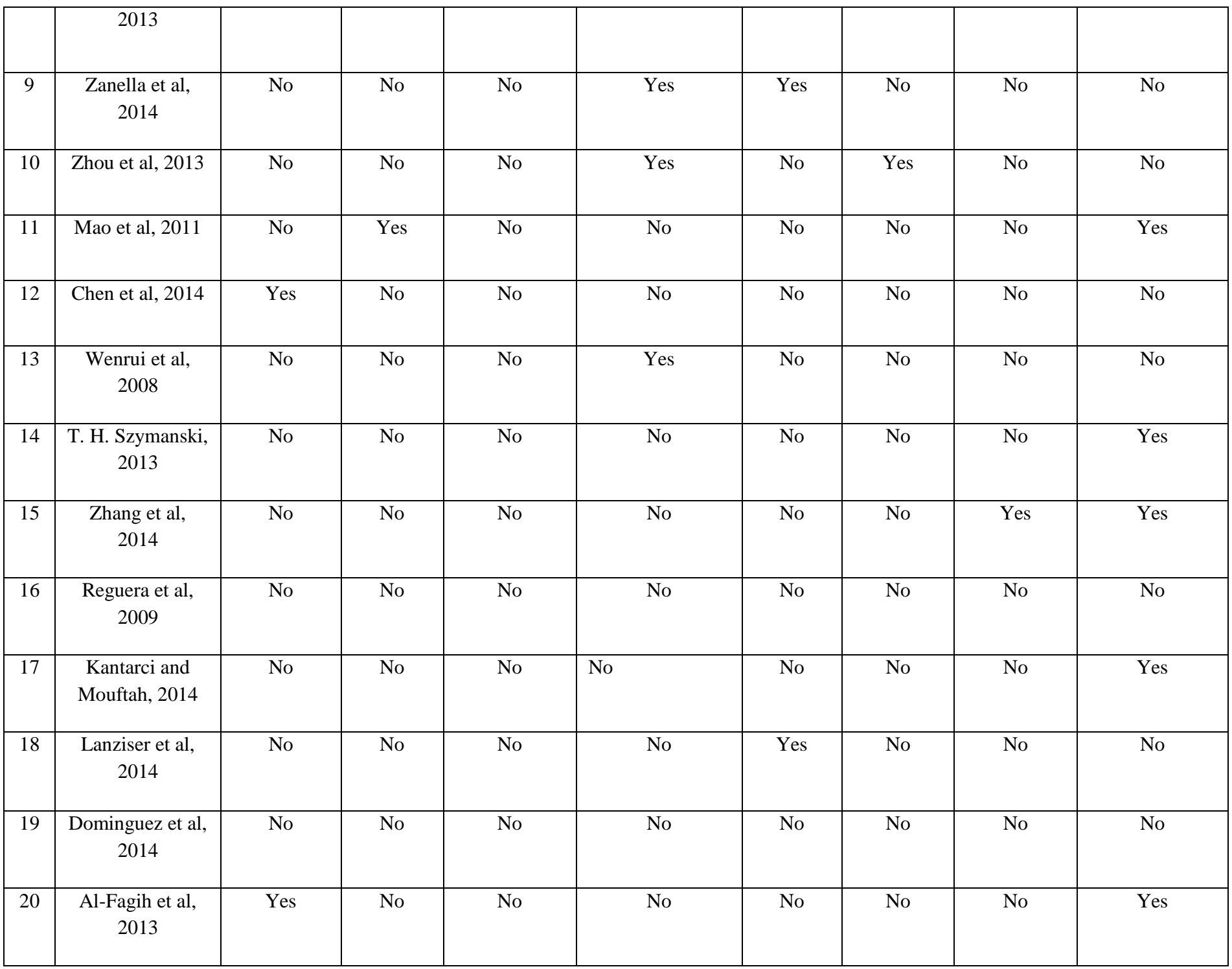

Table 3: Analysis of Quality Parameters

\begin{tabular}{|c|c|c|c|c|c|c|c|c|}
\hline S\# & Techniques & Verification & Validation & Performance & Effectiveness & Correctness & Compatibility & Cost Effectiveness \\
\hline 1 & Jie et al,2008 & No & No & Yes & Yes & No & No & Yes \\
\hline 2 & $\begin{array}{c}\text { Foody and } \\
\text { Boyd, 2013 }\end{array}$ & No & No & No & No & Yes & No \\
\hline 3 & $\begin{array}{c}\text { Kalayeh et al, } \\
2013\end{array}$ & No & No & No & No & No & No \\
\hline 4 & $\begin{array}{c}\text { Hu et al, 2013 } \\
\text { R. Bose, 2013 }\end{array}$ & No & No & Yes & Yes & No & No & No \\
\hline 6 & $\begin{array}{c}\text { Kiljander et al, } \\
2014\end{array}$ & No & No & No & No & No & Yes & Yes \\
\hline 7 & $\begin{array}{c}\text { Dellepiane and } \\
\text { Angiati, 2014 }\end{array}$ & No & No & No & No & No & No & No \\
\hline
\end{tabular}




\begin{tabular}{|c|c|c|c|c|c|c|c|c|}
\hline 8 & $\begin{array}{l}\text { Li and Xiong, } \\
2013\end{array}$ & No & No & Yes & No & No & No & No \\
\hline 9 & $\begin{array}{l}\text { Zanella et al, } \\
2014\end{array}$ & No & No & No & Yes & No & No & Yes \\
\hline 10 & Zhou et al, 2013 & No & No & No & No & No & Yes & No \\
\hline 11 & Mao et al, 2011 & No & No & No & No & Yes & No & No \\
\hline 12 & Chen et al, 2014 & No & No & No & Yes & No & No & No \\
\hline 13 & $\begin{array}{l}\text { Wenrui et al, } \\
2008\end{array}$ & No & Yes & No & No & Yes & Yes & No \\
\hline 14 & $\begin{array}{c}\text { T. H. } \\
\text { Szymanski, } \\
2013\end{array}$ & No & No & No & Yes & No & No & Yes \\
\hline 15 & $\begin{array}{l}\text { Zhang et al, } \\
2014\end{array}$ & No & No & Yes & No & No & No & No \\
\hline 16 & $\begin{array}{l}\text { Reguera et al, } \\
2009\end{array}$ & No & No & No & No & No & No & Yes \\
\hline 17 & $\begin{array}{c}\text { Kantarci and } \\
\text { Mouftah, } 2014\end{array}$ & No & No & No & $85 \%$ & No & Yes & No \\
\hline 18 & $\begin{array}{c}\text { Lanziser et al, } \\
2014\end{array}$ & No & No & Yes & No & No & No & No \\
\hline 19 & $\begin{array}{l}\text { Dominguez et } \\
\text { al, } 2014\end{array}$ & Yes & No & No & No & Yes & No & No \\
\hline 20 & $\begin{array}{l}\text { Al-Fagih et al, } \\
2013\end{array}$ & No & No & Yes & No & No & No & Yes \\
\hline
\end{tabular}

\section{ANALYSIS}

Table 1 inculcates the evaluation criteria which have been used for the analysis of techniques. Table 2 and table 3 provide the result of analysis. Research comprises the survey of twenty research papers. Fifteen parameters are used for the evaluation. Table 2 and table 3 show that security concerns regarding data mining and its techniques are not considered in any of the research paper. Validation is not discussed in any of the paper because the system is too complex to validate. Karpagavadivu [2], C. Seiffert [6], Z. Liaghat [8], S. Gupta [9], H. Nijadat [13], S. Gupta [14], D.J. Drown [15], L. Nussbaum [19] and C. Huang [20] address the performance attribute of the system. They refer to such techniques like genetic algorithm and clustering techniques applied on several systems which results in enhanced performance as well as effectiveness of the system. S. Gupta et al [9], D.V. Ashoka et al[12] and $\mathrm{H}$. Nijadat et al[13] address the reusability of work in the future for the new software development process. D.V. Ashoka et al[12] discussed the portability is the consequence of reusability thereby enhancing the overall quality of the product. D.V. Ashoka et al [12] and L. Nussbaumi et al [19] addressed the integration of data for the purposes of both data mining as well as quality assurance. This is done by removing the inconsistencies from the data in order to simplify the model. D.V. Ashoka et al [12] and S.Gupta et al [14] discussed that reusability enhanced the productivity of the product thereby enhancing the overall quality of the end product. S. Sharma et al [3] , N. Azeem [5], Z. Liaghat [8], D. Port et a[16] and L. Tuovinen et al[17] discussed that data mining and its techniques help in considerable reduction in cost. Sharma et al [3] showed that using an automated testing model to support the activities of human tester can reduce the cost of the testing process and related maintenance costs. B. Turhan et al [1], H. Nijadat et al [13] and S.Gupta et al[14] discussed that predicting fault prone modules earlier in the development help the software managers to allocate more resources to such modules. Resources constitute both testing as well as human resources. Maintainability is the technique which is being followed in most of the papers including S. Sharma et al[3], P. J. Kaur et al[4], N. Azeem et al[11], D.V. Ashoka et al[12] and H.Nijadat, et al[13], .The technique which is trending among all researches is extendibility. All researches have the capability to adapt new features to improve the system for the 
better quality. No time or deadline is mentioned in which the product is to be completed.

Efficiency of the system is considered in some papers in the following ways. Sharma et al [3] showed that using automated testing models improve the efficiency of the system by reducing the time taken by the manual testing process. $\mathrm{Z}$. Liaghat [8] and P. J. Kaur [4] showed that testing and system efficiency can be improved by a timely identification of faultprone modules. L. Wu [10] presented that Neutral Online Visualization-aided Autonomic evaluation framework (NOVA) framework improves the system efficiency as it is able of evaluating the real-time online machine learning and data mining.

S. Sharma et al [3] evaluated the effectiveness of the IFN (Info-Fuzzy Network) methodology on a complex expertsystem application having multiple continuous outputs while Karpagavadivu [2] improved the effectiveness of RIDOR (RIpple DOwn Rule) algorithm. Karpagavadivu [2] provided accuracy in fault prediction using $\mathrm{K}$ - Means based techniques for classification of software modules into faulty or non-faulty module. $\mathrm{L}$. Wu [10] worked to improve the effectiveness of system through different sampling techniques.

Reliability is discussed in N. Azeem [5], S. Zhang [7], S. Gupta [9], L. Wu [10], N. Azeem et al [11] and H. Nijadat, et al [13]. They used techniques like complexity metrics and NOVA for increasing the reliability of the system. B. Turhan et al [1] and S. Sharma et al [3] discussed different factors for improving the software testing. B. Turhan et al [1] showed that early detection of defects helps the software managers in prioritizing software testing, and call graph based ranking (CGBR) framework decreases the required testing effort for manual inspection of the source code and S. Sharma et al [3] focused on reducing the cost of the testing process through automated testing models. N. Azeem [5], S. Zhang [7], H. Nijadat, et al [13] and R. Feldt [18] worked on complexity reduction through different data mining techniques such as product metrics and complexity metrics.

This is analyzed that if the amount of previously available data is limited, system would not be assessable. There are some techniques, being used in different researches, which take data on random basis and then generate the results. It does not affect the performance of the system but may cause the difference in the results.

\section{CONCLUSION}

This research has evaluated IoT implementation techniques against quality assurance parameters. The quality assurance in IoT is a new era of research. In this paper we have surveyed various techniques for the implementation of IoT. We have evaluated and analyzed the criterion for comparison of defined techniques. From our survey, we conclude that most of the techniques lack tool support and automation techniques. Therefore, we suggest that there must be a generic methodology to implement IoT. A highly generic method must be customizable to specific domain. Following generalization will encourage tool support for IoT in its implementation.

\section{REFERENCES}

[1] S. Jie, S. Peiji, and F. Jiaming, "Effect of incentives on web-based surveys", Tsinghua Sci. Technol., vol. 13, no. 3, pp. 344-347, Jun. 2008.
[2] G. M. Foody, and D. S. Boyd, "Using volunteered data in land cover map validation: Mapping West African forests",IEEE J. Sel. Topics Appl. Earth Observ. , vol. 6, no. 3, pp. 1305-1312, Jun. 2013.

[3] M. M. Kalayeh, T. Marin, and J. G. Brankov, "Generalization evaluation of machine learning numerical observers for image quality assessment", IEEE Trans. Nucl. Sci, vol. 60, no. 3, pp. 1609-1618, Jun. 2013.

[4] L. Hu, Z. Zhang, F. Wang, and K. Zhao, "Optimization of the deployment of temperature nodes based on linear programming in the internet of things", Tsinghua Sci. Technol.,vol. 18, no. 3, pp. 250-258, Jun. 2013.

[5] R. Bose, "A paradigm shift in internet-based remote experimentation", IEEE access, vol. 1, pp. $718-725$, Oct. 2013.

[6] J. Kiljander et al., "Semantic interoperability architecture for pervasive computing and internet of things", IEEE access, vol. 2, pp. 853-873, Aug. 2014.

[7] S. G. Dellepianeand E. Angiati, "Quality assessment of despeckled SAR images", IEEE J. Sel. Topics Appl. Earth Observ., vol. 7, no. 2, pp. 691-707, Feb. 2014.

[8] F. Li and P. Xiong, "Practical secure communication for integrating wireless sensor networks into the internet of things", IEEE sensors J., vol. 13, no. 10, pp. 3677-3684, Oct. 2013.

[9] A. Zanella, N. Bui, A. Castellani, L. Vangelista, and M. Zorzi, "Internet of things for smart cities", IEEE internet things J., vol. 1, no. 1, pp. 22-32, Feb. 2014.

[10] Jin Zhou, Liang Hu, Feng Wang, Huimin Lu, and Kuo Zhao, "An efficient multi-dimensional fusion algorithm for IoT data based on partitioning", Tsinghua Sci. Technol., vol. 18, no. 4, pp. 369-378, Aug. 2013.

[11] X. Mao, C. Zhou, Y. He, Z. Yang, S. Tang, and W. Wang, "Guest editorial: Special issue on wireless sensor networks, cyber-physical systems, and internet of things", Tsinghua Sci. Technol., vol. 16, no. 6,pp. 559560, Dec. 2011

[12] S. Chen, H. Xu, D. Liu, B. Hu, and H. Wang, "A vision of IoT: Applications, challenges, and opportunities with china perspective", IEEE internet things J., vol. 1, no. 4, pp. 349-359, Aug. 2014

[13] D. Wenrui, T. Yubing, Z. Qishan , and Y. Dongkai, "Image and video quality assessment using neural network and SVM", Tsinghua Sci.Technol., vol. 13, no. 1, pp. 112-116, Feb. 2008.

[14] T. H. Szymanski, "Max-flow min-cost routing in a future-internet with improved QoS guarantees", IEEE Trans. Commun., vol. 61, no. 4, pp. 1485-1495, Apr. 2013.

[15] Y. Zhang, Z. Zheng, and M. R. Lyu, "An online performance prediction framework for service-oriented systems", IEEE Trans. Syst., Man, Cybern., Syst., vol. 44, no. 9, pp. 1169-1181, Sep. 2014.

[16] V. A. Reguera, E. M. G. Fernández, F. A. Paliza, W. Godoy Jr., "VoIP quality of service in networks with active queue management", IEEE latin America Trans., vol. 7, no. 5, pp. 603-611, Sep. 2009. 
[17] B. Kantarci and H. T. Mouftah, "Trustworthy sensing for public safety in cloud-centric internet of things", IEEE internet things J., vol. 1, no. 4, pp. 360-368, Aug. 2014.

[18] S. Lanziser, A. R. Weber, A. Liao, D. Pajak, and A. K. Meier, "Communicating power supplies: Bringing the internet to the ubiquitous energy gateways of electronic devices", IEEE internet things J., vol. 1, no. 2, pp. 153160, Apr. 2014.
[19] L. R. Dominguez, D. M. Rodriguez, C. V. Rosales, D. T. Roman, and J. R. Pacheco, "RTT Prediction in Heavy Tailed Networks", IEEE CommunLett., vol. 18, no. 4, pp. 700-703, Apr. 2014.

[20] A. E. Al-Fagih, F. M. al-Turjman, W. M. Alsalih, AND H. S. Hassanein, "A Priced Public SensingFramework for Heterogeneous IoT Architectures", IEEE Trans. Emerg. Topics Comput., vol. 1 , no. 1, pp. 133-147, Aug. 2011. 